NBER WORKING PAPER SERIES

\title{
THE (NON-) EFFECT OF OPPORTUNITY ZONES ON HOUSING PRICES
}

\author{
Jiafeng Chen \\ Edward L. Glaeser \\ David Wessel \\ Working Paper 26587 \\ http://www.nber.org/papers/w26587 \\ NATIONAL BUREAU OF ECONOMIC RESEARCH \\ 1050 Massachusetts Avenue \\ Cambridge, MA 02138 \\ December 2019
}

We thank Isaiah Andrews, Ashesh Rambachan, Jonathan Roth, Suproteem Sarkar, Liyang Sun, and Christopher Walker for helpful discussions. We especially thank Yanchen Jiang for superb research assistance. All errors are our own. The views expressed herein are those of the authors and do not necessarily reflect the views of the National Bureau of Economic Research.

At least one co-author has disclosed a financial relationship of potential relevance for this research. Further information is available online at http://www.nber.org/papers/w26587.ack

NBER working papers are circulated for discussion and comment purposes. They have not been peer-reviewed or been subject to the review by the NBER Board of Directors that accompanies official NBER publications.

(C) 2019 by Jiafeng Chen, Edward L. Glaeser, and David Wessel. All rights reserved. Short sections of text, not to exceed two paragraphs, may be quoted without explicit permission provided that full credit, including ( $)$ notice, is given to the source. 
The (Non-) Effect of Opportunity Zones on Housing Prices

Jiafeng Chen, Edward L. Glaeser, and David Wessel

NBER Working Paper No. 26587

December 2019

JEL No. H73,R30,R38,R58

\section{ABSTRACT}

Will the Opportunity Zone program, America's largest new place-based policy in decades, generate neighborhood change? We compare single-family housing price growth in Opportunity Zones with price growth in areas that were eligible but not included in the program. We also compare Opportunity Zones to their nearest geographic neighbors. All estimates rule out price impacts greater than 1.3 percentage points with $95 \%$ confidence, suggesting that, so far, home buyers don't believe that this subsidy will generate major neighborhood change. Opportunity Zone status reduces prices in areas with little employment, perhaps because buyers think that subsidizing new investment will increase housing supply.

Jiafeng Chen

Department of Economics

Littauer Center

1805 Cambridge Street

Cambridge, MA 02138

jiafengchen@g.harvard.edu

Edward L. Glaeser

Department of Economics

315A Littauer Center

Harvard University

Cambridge, MA 02138

and NBER

eglaeser@harvard.edu
David Wessel

Brookings Institution

and Wall Street Journal

dwessel@brookings.edu 


\section{Introduction}

Opportunity Zones, established by the Tax Cuts and Jobs Act of 2017, arguably represent the U.S. government's largest place-based policy innovation since Empowerment Zones were introduced in 1993. When capital gains are invested in Opportunity Zones, taxes on the original gains are deferred and can be reduced; taxes on future gains from qualifying investments in Opportunity Zones are largely eliminated. Will this significant tax-based subsidy lead to neighborhood improvements?

We test this hypothesis by examining whether areas that are designated as Opportunity Zones in mid-2018 subsequently experience an increase in residential real estate prices after 2017. If Opportunity Zone designation is seen as a harbinger of future investment and upgrading, then new buyers should presumably anticipate future neighborhood improvements and be willing to pay more for homes. If Opportunity Zones are ineffective, or act primarily to generate more residential supply, then Opportunity Zones will have little impact on price. It is too early to test the more interesting and important question of whether Opportunity Zones impact people as well as place, and whether they positively impact the lives of neighborhood residents. ${ }^{1}$ Moreover, we only have price data available for 2018, and the Opportunity Zone tracts were designated by governors in the first few months of 2018 and officially posted in July. ${ }^{2}$

A non-academic study of housing prices, done by Zillow (Casey, 2019), found a positive effect of Opportunity Zones on prices when comparing Opportunity Zone areas with areas that did not receive zone status, but there are two reasons to be cautious about this work. First, Zillow's price data reflects an opaque algorithm, rather than actual sales data. Second, the pre-2017 trends in prices between their treatment and control samples do not appear to be parallel, although they provide no tested pre-trends. Third, Casey (2019) results also do not control for changes in the quality of houses sold, since they include all arms' length sales prices.

We use Federal Housing Finance Agency (FHFA) repeat sales-indices for single-family homes at the Census tract and ZIP code level to measure price changes. We perform three different empirical exercises. First, we follow the Zillow study (Casey, 2019) and compare Opportunity Zone areas with areas that were initially eligible for Opportunity Zone status, but then not included as Opportunity Zones, assuming and testing for parallel trends. Second, we use propensity-score weighting methods to include observed characteristics nonparametrically in the difference-in-difference design, making and testing a conditional parallel trends assumption. Third, we compare Opportunity Zone areas with bordering areas. As many tracts have missing data, we perform the exercises at the ZIP code level in addition to at the Census tract level as well.

All exercises yield a similar result: Opportunity Zones appear to have a negligible price impact that is statistically indistinct from zero. Our results are sufficiently precise that we can generally rule

\footnotetext{
${ }^{1}$ Busso and Kline (2008) provide a thorough analysis of the economic impact of national employment zones, which appear to have meaningfully impacts both housing prices and employment. Neumark and Kolko (2010) find fewer positive effects of state level Enterprise Zones.

${ }^{2}$ Consequently, only one-half of the year should be counted as treated by the program. We can do nothing to extend the analysis temporally until more data is released, and we think that a reasonable interpretation is to double the estimated coefficients to reflect the fact that only one-half of the year was fully treated by the policy.
} 
out a price impact of 1.3 percentage points or more at the tract level with $95 \%$ confidence. Our point estimates are typically between 0.3 and -0.5 percentage points. This finding suggests that buyers do not believe that Opportunity Zone status will generate a significant change in the economic fortunes of the neighborhood. Alternatively, buyers could be myopic, but that seems unlikely if the zone status attracts professional investors. One possibility is that this null result reflects a combination of a positive demand shock and positive housing supply shock, since Opportunity Zone status might encourage more residential construction. To test this possibility, we compare ZIP codes that are predominantly residential with ZIP codes that are predominantly commercial. The positive impact of Opportunity Zones on housing supply should be stronger in non-commercial areas, yet we find that Zone status has an essentially identical impact in commercial and residential areas.

We find that Opportunity Zone status is associated with price declines in highly residential areas. In the treated ZIP codes with the lowest share of employment to residences, as the share of households that lies within an opportunity zone increases from 0 to 100 percent, prices fall by 1.7 percentage points. In areas with more employment, the impact of Opportunity Zone status is generally positive. The highest point estimate in regressions that include time-varying coefficients on covariates is 1.1, meaning that varying from no Opportunity Zone households to 100 percent Opportunity Zone households is associated with 1.1 percentage points higher housing prices at the ZIP level.

This work does not imply that Opportunity Zones were a mistake or that there are no benefits from these zones. These tax subsidies may generate neighborhood change in the future that is not anticipated by buyers today. The costs of these subsidies may end up being so small that they are offset by even tiny price gains. Nonetheless, the absence of a visible price effect does suggest the limits of place-based policies, especially those that focus on investment in physical rather than human capital.

This paper proceeds as follows. Section 2 discusses the institutional context, policy details, and selection procedures for Opportunity Zones. Section 3 discusses the various data sources used in our study. Section 4 introduces our three main empirical strategies. Section 5 discusses the empirical results. Section 6 concludes.

\section{Institutional Context}

Opportunity Zones were created in December 22, 2017, when President Trump signed the Tax Cuts and Jobs Act (Public Law 115-97, henceforth TCJA) into law. They are intended to spur economic development in distressed communities. The law provides three benefits for investing capital gains in one of 8,762 Census tracts ${ }^{3}$ ( $12 \%$ of all Census tracts) across the country through intermediaries called Opportunity Funds: Tax on the initial capital gain is deferred until 2026 or when the asset is sold. For capital gains placed in an Opportunity Fund for at least five years, investors' basis

\footnotetext{
${ }^{3}$ Two additional tracts in Puerto Rico were added by subsequent legislation, bringing the total to 8,764. We do not have housing data for Puerto Rico.
} 
on the original investment increases by $10 \%$; if invested for seven years, by $15 \% .{ }^{4}$ For investments in Opportunity Funds held for at least 10 years, the gains on the investments in the zones are not subject to capital gains tax. Funds can be invested in commercial, residential and industrial real estate; infrastructure, and businesses. For real estate projects to qualify, the investment must result in the property being "substantially improved."

The outlines of a proposal to create Opportunity Zones was published by the Economic Innovation Group in April 2015. ${ }^{5}$ Bills to create them were first introduced in Congress in April 2016 and re-introduced in February 2017, but got little attention initially. As in the bill that eventually became law, the proposals authorized governors to nominate as Opportunity Zones 25\% of the "low income communities" in their states; in states with fewer than 100 low income communities, the governor could choose 25. The definition of low income communities was borrowed from a 2000 law that created the New Markets Tax Credit: Census tracts were designated as low income if the poverty rate is at least $20 \%$, or the median family income doesn't exceed $80 \%$ of the statewide median for a tract outside the metropolitan area, or the median income doesn't exceed $80 \%$ of the statewide median or the metro area statewide median for a tract inside a metropolitan area. Tracts contiguous with low income communities also are eligible, provided their median family income doesn't exceed $125 \%$ of the contiguous low income community.

The Opportunity Zone provision was not included in the House version of the TCJA, which was introduced on Nov. 2, 2017. With very little public attention, Sen. Tim Scott (R, S.C.), a member of the Senate Finance Committee, successfully pushed to include the Opportunity Zone provision in the TCJA, which was introduced on Nov. 28, 2017. The first reference in the press to the Opportunity Zone provision in the TCJA came on November 28, 2017, in South Carolina's Post and Courier, according to the Factiva database. ${ }^{6}$

Governors had 90 days after the passage of the law - until March 21, 2018 unless they sought a 30-day extension - to nominate zones from a list of 31,866 eligible Census tracts prepared by the Treasury based on 2010-2015 American Community Survey data. The Treasury posted a list of all qualified Opportunity Zones on July 9, 2018. Of the 8,762 Opportunity Zones, 8,534 are low income communities and 230 are contiguous Census tracts. A map of the zones in the U.S. mainland is shown in Figure 1.

\section{Data}

Our measure of housing price growth is the annual change in the housing price index computed by the Federal Housing Finance Agency (FHFA). The housing price index is a weighted, repeatsales price index of the movement of single-family house prices. Like all repeated-sales indices, it

\footnotetext{
${ }^{4}$ The $10 \%$ step-up in basis is only for Opportunity Zone investments made by Dec. 31, 2021. The additional $5 \%$ step-up in basis is for investments by Dec. 31, 2019.

${ }^{5}$ https://eig.org/wp-content/uploads/2015/04/Unlocking-Private-Capital-to-Facilitate-Growth.pdf

${ }^{6}$ https://www. postandcourier.com/politics/sens-scott-graham-are-big-arm-twisters-in-tax-overhaul/ article_3e414eb2-d453-11e7-9ea8-93ddcd71dd65. html
} 
attempts to correct for quality changes. We use the annual house price indices by Census tract and by five-digit ZIP codes, ${ }^{7}$ and treat 2018 as the treated year.

Information about the Opportunity Zones is provided by the Urban Institute. ${ }^{8}$ The data includes whether a tract belongs to the 31,866 eligible tracts and to the selected 8,762 tracts and whether a tract is eligible for selection as a low income community or as contiguous Census tracts, which we use in our first and second empirical designs in Section 4. Characteristics of the Census tracts are from the American Community Survey (ACS) 2013-2017 5-year estimates.

Geographical comparison between tracts and their non-selected geographical neighbors uses the TIGER 2018 geographic shapefiles provided by the Census. ${ }^{9}$ Aggregating tract-level data to ZIPlevel data, implemented in Section 4.4, uses the geographical crosswalks between 5-digit USPS ZIP codes and Census tracts are provided by the Office of Policy Development and Research at the Department of Housing and Urban Development, where we use the data for the first quarter of 2017. ${ }^{10}$ Lastly, splitting ZIP codes by employment population in Table 2 uses population data at the ZCTA level in the 2012-2016 ACS 5-year estimates and employment data from the ZIP code level County Business Patterns in 2016. ${ }^{11}$

\section{Empirical Strategy}

We use three main empirical strategies. First, following Casey (2019), we compare Opportunity Zones to Census tracts that are eligible for Opportunity Zones but are not selected. ${ }^{12}$ In this case, we use a difference-in-differences design that optionally incorporates observable tract-level covariates interacted with year fixed effects. We supplement the analysis in Casey (2019) with formal tests of pre-treatment trends. Second, we refine our analysis in the first design with semiparametric propensity score weighting methods in a difference-in-differences setting (Abadie, 2005; Callaway and Sant'Anna, 2018; Sant'Anna and Zhao, 2018). Third, we compare Opportunity Zones with their geographical neighbors that are not selected.

The FHFA tract-level data covers only about half of the selected Census tracts. Concerned with the data attrition, we also aggregate tract-level data to the ZIP code level, and uses FHFA data at the ZIP code level, which has better coverage. ${ }^{13}$ Lastly, we split the ZIP-code level data into quartiles by employment population so as to decompose the potential effects of Opportunity Zones on supply and demand, noting that the positive supply effect has larger impact in residential

\footnotetext{
${ }^{7}$ https://www. fhfa.gov/DataTools/Downloads/Pages/House-Price-Index-Datasets. aspx

${ }^{8}$ https://www.urban.org/policy-centers/metropolitan-housing-and-communities-policy-center/projects/ opportunity-zones

${ }^{9}$ https://www. census.gov/geographies/mapping-files/time-series/geo/tiger-line-file.html

${ }^{10}$ https://www. huduser.gov/portal/datasets/usps_crosswalk.html

${ }^{11}$ https://www. census.gov/programs-surveys/cbp.html

${ }^{12}$ In the results presented in the main text, we compare selected tracts to tracts that are eligible but not selected conditional on the eligibility criterion being low-income community, as tracts that are eligible for contiguity reasons are overrepresented in the non-selected group. Qualitative conclusions do not change if we remove this condition (Appendix C).

${ }^{13}$ Although only 16,007 of the total 39,300 ZIP codes with crosswalk data do not have missing data in 2018, these ZIP codes intersect with 6,854 selected Opportunity Zones.
} 
areas.

Let $i$ denote Census tracts and let $t=1, \ldots, T$ denote time periods. Treatment occurs in time $T$. For each Census tract $i$, we observe annualized housing price growth $Y_{i t}^{\text {obs }}$ and treatment status $D_{i t}=\mathbb{1}(t=T) D_{i}$ along with a vector of covariates $X_{i}$. The potential outcomes are $Y_{i t}(1)$ and $Y_{i t}(0)$ with $Y_{i t}^{\text {obs }}=D_{i t} Y_{i t}(1)+\left(1-D_{i t}\right) Y_{i t}(0)$. We are interested in the estimand

$$
\tau=\mathbb{E}\left[Y_{i T}(1)-Y_{i T}(0) \mid D_{i}=1\right]
$$

which is the average treatment effect on the treated (ATT). Assume we have a balanced panel where $Z_{i}=\left(Y_{i}, X_{i}, D_{i}\right)$ are independently and identically distributed.

\subsection{Baseline difference-in-differences (Casey, 2019)}

In the first design, we compare tracts that are selected as Opportunity Zones to tracts that are eligible but not selected. The parallel trends assumption allows us to identify the treatment effect in a difference-in-differences design. As we see in Figure 3, parallel trends is a more plausible assumption when comparing selected tracts to eligible but not selected tracts than when comparing selected tracts to all other tracts.

Our first estimation strategy specifies a model where we allow for individual-specific heterogeneity in levels and overall trends (Angrist and Pischke, 2008) via two-way fixed effects: $Y_{i t}(0)=\mu_{i}+\alpha_{t}+\epsilon_{i t}^{0}$ and $Y_{i t}(1)=\mu_{i}+\alpha_{t}+\tau+\epsilon_{i t}^{1}$ with $\mathbb{E}\left[\epsilon_{i t}^{0} \mid D_{i}\right]=\mathbb{E}\left[\epsilon_{i t}^{1} \mid D_{i}\right]=0 .{ }^{14}$ The usual parallel trends assumption is implied by the model, since $\mathbb{E}\left[Y_{i t}(0)-Y_{i, t-1}(0) \mid D_{i}=d\right]=\alpha_{t}-\alpha_{t-1}$, which does not depend on treatment status $d$. Thus $\tau$ is consistently estimated by $\widehat{\tau}$ in the OLS regression

$$
Y_{i t}^{\mathrm{obs}}=\mu_{i}+\alpha_{t}+\tau \mathbb{1}\left(t=T, D_{i}=1\right)+\epsilon_{i t}
$$

Column (1) in the top panel of Table 1 implements this design, clustering standard errors at the tract level. ${ }^{15}$ We also adapt the two-way fixed effect model (2) by including covariates, replacing $\alpha_{t}$ with $\alpha_{i t}=X_{i}^{\prime} \alpha_{t}$ for a vector of covariates $X_{i}$. This specification assumes conditional parallel trends, as $\mathbb{E}\left[Y_{i t}(0)-Y_{i, t-1}(0) \mid X_{i}=x, D_{i}=d\right]=x^{\prime}\left(\alpha_{t}-\alpha_{t-1}\right)$, is free of the treatment status $d$. Column (2) in the top panel of Table 1 implements this design, where the test against pre-trends is defined analogously.

\subsection{Propensity-score-weighted difference-in-differences}

Identification using the selection of Opportunity Zones from eligible Census tracts faces the challenge that, selected tracts and tracts that are eligible but not selected differ in observable char-

\footnotetext{
${ }^{14}$ The model assumes constant treatment effects. When treatment effects are heterogeneous, the two-way fixed effect estimator is consistent for a weighted average of individual treatment effects.

${ }^{15}$ We can test against the identification assumption by testing aginst against the hypothesis $\lambda_{t}=0$ for all $t<T$ in the regression

$$
Y_{i t}^{\mathrm{obs}}=\mu_{i}+\alpha_{t}+\lambda_{t} D_{i}+\delta_{i t} .
$$
}

The coefficients $\lambda_{t}$ are plotted in an event-study plot in Figure 3 . 
acteristics. Unbalanced characteristics suggests that parallel trends may not hold. ${ }^{16}$ We alleviate these concerns by incorporating observed covariates in a nonparametric fashion, in our second empirical strategy. We use an adaptation of propensity score weighting estimator introduced by Abadie (2005). In Abadie (2005), the probability of treatment is nonparametrically estimated as a function of the covariates; the estimated propensity scores are then used to form an inverse propensity weighting estimator of the ATT. Recent work by Callaway and Sant'Anna (2018) extends this approach to settings with multiple periods, multiple treatment groups, and multiple treatment timings. ${ }^{17}$ We apply the Callaway and Sant'Anna (2018) approach in Column (3) of the top panel of Table 1. Sant'Anna and Zhao (2018) extend Abadie (2005)'s two-period, two-group model and introduces a doubly-robust version of the semiparametric estimator in Abadie (2005). The ATT estimator is consistent if either the propensity score model or the outcome regression model is correctly specified. Column (4) in the top panel of Table 1 implements this approach with a balanced panel from 2017-2018.

\subsection{Comparison of geographical neighbors}

Our third empirical strategy uses geographic proximity to construct a paired sample of tracts. For each selected tract $i$, we construct its non-selected neighbor $\tilde{i}$ to be the tract that is (i) not selected as an Opportunity Zone, (ii) closest to $i$ by distance between centroids, (iii) in the same state as $i$, and (iv) has no missing housing price data in 2018. ${ }^{18}$ Within each pair $\iota=(i, \tilde{i})$, we specify $Y_{i t}(0)=\gamma_{\iota}+\alpha_{\iota t}+\epsilon_{\iota t}, Y_{\tilde{i} t}(0)=\tilde{\gamma}_{\iota}+\alpha_{\iota t}+\tilde{\epsilon}_{\iota t}$ such that $Y_{i t}(0)-Y_{i t}(0)=\left(\gamma_{\iota}-\tilde{\gamma}_{\iota}\right)+\left(\epsilon_{\iota t}-\tilde{\epsilon}_{\iota t}\right)$, leading to the estimation procedure

$$
Y_{i t}^{\mathrm{obs}}-Y_{\tilde{i} t}^{\mathrm{obs}}=\tau \mathbb{1}\left(t=T, D_{i}=1\right)+\mu_{\iota}+\eta_{\iota t},
$$

which is consistent assuming $\mathbb{E}\left[\eta_{\iota t} \mid D_{i}\right]=0$. The identification assumption in the third strategy is the pair, $i, \tilde{i}$ has the same trend $\alpha_{\iota t}$, which is differenced away as we construct the estimator. We implement this approach in Column (5) in the top panel of Table 1, where an additional linear trend is included in Column (6). We also plot the mean paired differences in Figure 3, normalizing the difference in 2017 to zero.

\footnotetext{
${ }^{16}$ We do find substantial imbalance in covariate values in Tables 4 and 5 in the Appendix. Technically, the identification assumption, (conditional) parallel trends, does not require covariate balance, since trends are only required to be parallel and may differ in levels.

${ }^{17}$ In our design, we only have multiple pre-periods. There is a recent literature on the failure of the two-way fixed effect estimator (2) in situations with variable treatment timing and heterogeneous treatment effects, as the estimator becomes a weighted average of individual treatment effects with non-convex weights in large samples (Abraham and Sun, 2018; Athey and Imbens, 2018; Callaway and Sant'Anna, 2018; Goodman-Bacon, 2018; Imai and Kim, 2016; Borusyak and Jaravel, 2017; De Chaisemartin and D'Haultfœuille, 2017). The issue is not as pertinent in our setting as we do not have variable treatment timing.

${ }^{18}$ The distance between centroids is calculated using the Haversine formula, assuming the Earth is a sphere with radius 6,371 kilometers. The average centroid distance between pairs is 2.718 (5.724) kilometers.
} 


\subsection{Aggregating to ZIP codes}

The FHFA tract-level data only covers half of all treated Opportunity Zones. Moreover, the sample suffers from further attrition due to panel balance and missing Census covariates. To address the data availability concern, we include an alternative design by aggregating tracts to the ZIP code level. Mimicking our tract-level analysis, we drop ZIP codes that do not intersect with any Census tracts that are eligible to be selected as Opportunity Zones. ${ }^{19}$ Each ZIP code $z$ is partitioned into tracts $i \in I_{z}$, with $\pi_{i}^{z}$ proportion of total addresses within tract $i$. We choose this aggregation method because crosswalks are readily provided by the Department of Housing and Urban Development and addresses are the most relevant measure for residential housing prices. For

each variable $V$, we construct $V_{z}=\sum_{i \in I_{z}} \pi_{i}^{z} V_{i}$. The ZIP-code level treatment exposure $D_{z}$ now has a continuous distribution on $[0,1]$.

We report estimates after aggregation to the ZIP level in the bottom panel of Table 1. The strategy in (2) extends to this setting, which is implemented in Columns (1) and (2) of the bottom panel of Table 1. We discretize $D_{z}$ for weighting based estimator in Sant'Anna and Zhao (2018), by taking $\tilde{D}_{z}=\mathbb{1}\left(D_{z} \geq q\right)$ where $q$ is chosen such that the sample mean of $\tilde{D}_{z}$ equals the proportion of treated tracts among all tracts. The Sant'Anna and Zhao (2018) estimator is implemented in Column (3) in the bottom panel of Table 1.

As a refinement of the ZIP-code level analysis, we use data from County Business Patterns and split our sample on quartiles of the ratio between population employed in the ZIP code to population residing in the ZIP code ${ }^{20}$ and perform the analysis of Columns (1) and (2) in Table 1 separately for each quartile. ${ }^{21}$ The results are presented in Table 2 and discussed in Section 5.1.

\section{Results}

The top panel of Table 1 provides tract-level results, corresponding to empirical strategies in Sections 4.1 and 4.3. The bottom panel shows ZIP code level results corresponding to Section 4.4. As discussed above, the key independent variable in the tract level regressions is an indicator variable that takes on a value of one if the tract is designated an Opportunity Zone. In the ZIP code level regression, the key independent variable is the share of the addresses within each ZIP code that lie within an Opportunity Zone.

The first two regressions in Table 1 show results where the treated tracts are compared with tracts that were eligible for inclusion within Opportunity Zones, but were ultimately not included in the Zones. These results include time and tract fixed effects, and the estimated coefficient is 0.248 , meaning that prices rose by about one-fourth of a percentage point in tracts that were included in Opportunity Zones in 2018. This coefficient is small in magnitude, statistically insignificant and precisely enough estimated so that we can rule out an effect of more than 0.7 percentage points.

\footnotetext{
${ }^{19}$ Our empirical results are not sensitive to this choice.

${ }^{20}$ This ratio can exceed 1 if the ZIP code is more commercial than residential.

${ }^{21}$ The quartile thresholds are $0.134,0.244,0.409$ in the employment to population ratio.
} 
In the second column, we show results allowing for interactions between tract-level characteristics and year, so that we estimate coefficients on tract income and other characteristics for each year. The tract level covariates do not change over time. With these added controls, the coefficient falls to 0.151. Again, the coefficient is small and insignificant. In this case, we can rule out effects of greater than 0.6 percentage points with $95 \%$ confidence.

Figure 2 shows the tract level housing price indices visually. The top two lines show annual growth rates from 2014 to 2018 for the treatment and control samples that are evaluated in the first two columns of Table 1. Both of these lines are quite distinct from the third line, which contains all of the tracts in the U.S. that were never eligible for Opportunity Zone status. The top two lines lie essentially on top of one another. Pre-trends appear to be quite similar for the two top groups and quite different from the third group, which supports the finding of the pre-trends test reported in Table 1. There is also no visual change after the law is enacted in 2018. Both before and after the law is enacted, price growth in the two groups appears to be almost exactly the same.

The bottom panel of Table 1 shows results for the ZIP code analysis. In this case, the estimated coefficient represents the impact of moving from having no Opportunity Zone tracts within the ZIP code to having 100 percent Opportunity Zone tracts within the ZIP code. ${ }^{22}$ If the benefits of Opportunity Zone status spill over to neighboring tracts, then we would expect this coefficient to be larger than the coefficient in the tract-level analysis. The coefficient in the first column is indeed relatively large in the entire table, and it suggests that as the share of households that live in Opportunity Zone tracts increase from zero to one, prices increased by 0.48 percentage points. As the standard errors suggest that the true coefficient could be as high as 1.2 , this coefficient might be economically meaningful.

Yet there are two reasons to be cautious. First, the pre-trend test $p$-value suggests that the parallel trends assumption may be violated. ZIP codes with a higher share of addresses residing in Opportunity Zones seem to diverging from ZIP codes with a lower share of such households prior to 2018. Moreover, when we allow for time varying effects of other tract level characteristics in the second column, the coefficient becomes negative. That second coefficient is estimated with enough precision to rule out a coefficient greater than 0.6 at conventional confidence levels. We interpret these results to suggest that the ZIP code level analysis also rules out large positive price impacts of Opportunity Zone status in 2018.

In the third column, we show the tract-level analyses using the Callaway and Sant'Anna (2018) propensity score weighting method. The coefficient estimate is 0.25 , and the upper bound on the confidence interval is 0.89 . The fourth regression shows the doubly-robust coefficient estimate that follows Sant'Anna and Zhao (2018). This coefficient is even smaller. The point estimate is .08 and the upper bound estimate is 0.73. The Callaway and Sant'Anna (2018) procedure tests for pre-trends, and we do not reject the null hypothesis that there is no pre-trend; the doubly-robust procedure (Sant'Anna and Zhao, 2018) follows a two-period model, and does not provide a pre-

\footnotetext{
${ }^{22}$ The average ZIP code has $13.3 \%$ of its addresses in a selected Opportunity Zone; the median ZIP code has $0.0 \%$; and the 75 th percentile has $19.7 \%$.
} 
trend test. In both cases, the results imply that Opportunity Zone status increased prices by less than one percentage point in 2018 related to previous years.

In regressions (5) and (6), we match Opportunity Zone Census tracts with the nearest Census tract that is not in an Opportunity Zone. In some cases, the Opportunity Zone tracts are matched with the same non-Opportunity Zone tract. In regression (5), we find a coefficient of 0.74, which is statistically significant at conventional levels, but the result is not robust to inclusion of a linear time trend in regression (6). The coefficient falls to 0.534 and the $95 \%$-confidence interval rules out a coefficient greater than 1.3 .

Figure 3 shows year-by-year results that correspond to regressions (1), (2) and (5). The first two sets of coefficients show no pre-trend, but only a small and statistically insignificant increase in price in 2018. The third set of coefficients shows a statistically significant price increase in 2018 , but also suggestions of violation of parallel trends in 2014. Our interpretation of these results is that Opportunity Zone tracts did experience a modest increase in price in 2018 relative to the nearest geographic neighbors, but that this could easily be a reflection of a pre-existing trend that started before that year.

Taken together, these results suggest that if Opportunity Zone status did generate a positive impact, that impact was quite small. There seems to be little possibility that home buyers anticipated that inclusion in an Opportunity Zone would have a dramatic impact on the character of the neighborhood. This fact does not imply that the Opportunity Zone program was a mistake, but rather that it is anticipated to have little effect on the neighborhood.

\subsection{Heterogeneous Impacts of Opportunity Zone Designation}

Opportunity Zone status confers subsidies to physical investment in a neighborhood. Such subsidies might have a different impact on housing prices if they largely work by subsidizing commercial space or if they largely work by subsidizing residential space. If a capital subsidy increases the presence of job-generating commercial properties, then standard urban theory predicts that the subsidy will increase residential prices. If the subsidy increases investment in residential properties, then the impact on housing prices could be negative.

Consider a subsidy that decreases the costs of adding residential density, but assume that existing homes must be bought to provide the land needed to build. If the new building generates supply but not externalities, then this should decrease the value of housing units. The value of lower density homes, which make up the bulk of the FHFA repeat sales properties, could still increase because they are providing land for future investment. If the new investment generates positive externalities, then there could be a positive price impact even if supply increases.

We test the hypothesis that Opportunity Zone status may actually decrease prices by boosting residential supply by splitting ZIP codes into quartiles based on the level of employment to population before 2017. We use County Business Pattern data on employment levels, and we do not have this data below the ZIP Code level. Our core assumption is that Opportunity Zone status will act primarily as a subsidy to commercial properties in the quartiles that initially have the 
highest levels of employment to population and that Zone status will act primarily as a subsidy to residential properties in the lowest quartile, where employment to population begins at a low level.

We test for this heterogeneity in Table 2. As in the first two regressions in Table 1, the first column includes no covariates. The second columns include fixed covariates that are allowed to have a different coefficient in each year. Our primary interest lies in the top panel, which includes those ZIP codes with the lowest employment to population ratios and consequently the areas in which the subsidy is likely to primarily produce more residential housing supply.

The first columns shows a coefficient of -1.1 . Controlling for covariates pushes the coefficient to -1.7 , which rejects zero at the $p=0.1$ level with values like -3.7 lying within the confidence bounds. These results suggest that Opportunity Zone status may well have meaningfully reduced housing prices in largely residential areas, presumably because home buyers anticipated that the capital subsidy would lead to a larger supply of new homes in the future.

The bottom three panels show a hodge-podge of coefficients. The second column of results, with covariate-time interactions, show a result that is close to zero for the second quartile, a positive coefficient that is over one for the third quartile and a negative coefficient that is close to minus one for the fourth quartile. None of the second column results are statistically significant at conventional

rejection levels. We interpret these results as implying that we are unable to meaningfully estimate treatment heterogeneity outside the areas with the lowest levels of employment.

The results in Table 2 do, however, support the view that capital subsidies that reduce the cost of building homes reduce the price of homes in largely residential areas. Once again, these results do not imply that the Opportunity Zone program was a mistake. Lower housing prices in these areas may be a social good. Yet there is little evidence to support the view that Opportunity Zone status generated the expectation, at least among home buyers, that these areas would transition from poverty to prosperity.

\section{Conclusion}

Opportunity Zones are America's most important new national spatial policy since the Empowerment Zone program began during the Clinton era. They are intended to spur investment in high poverty areas. The hope of this program is that it would generate neighborhood revival, yet we find little evidence to support this view at this early date. Housing prices may have gone up in Opportunity Zone areas after their enactment in 2018, but if they did the overall price impact seems to have been less than one percentage point. In highly residential areas, Opportunity Zone status seems to have more meaningfully reduced residential home prices, presumably because Zone status generated a subsidy for building new homes.

The designation of the Opportunity Zone tracts was only made public in the summer of 2018. Consequently, our results reflect a mixture of treated and non-treated months, and an observer could reasonably double our estimated coefficients to form a posterior belief of the treatment effect of opportunity zone status. Moreover, the early nature of these results should make us cautious 
about any interpretation. We are at an early point and home price effects can, at best, capture the expectations about neighborhood change held by current home buyers. These buyers could be wrong: In the future Opportunity Zone status could end up correlated with neighborhood upgrading. Moreover, we have not evaluated the cost of the Opportunity Zone program, and so we can say nothing about overall program evaluation. Still, these early results do make us wonder whether America's troubled areas most need capital subsidies, or whether instead they need investments in human capital and neighborhood amenities. 


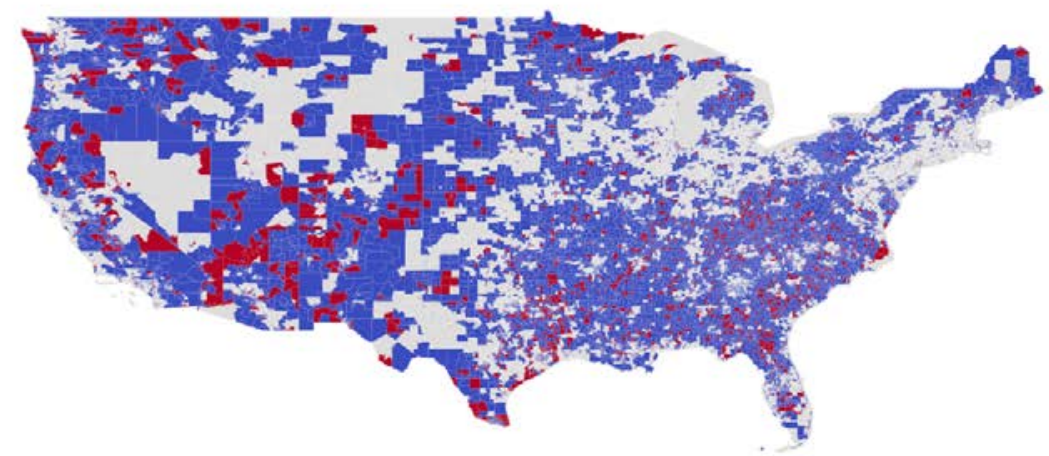

Eligible, not selected Ineligible

- Selected

Figure 1: Opportunity Zones

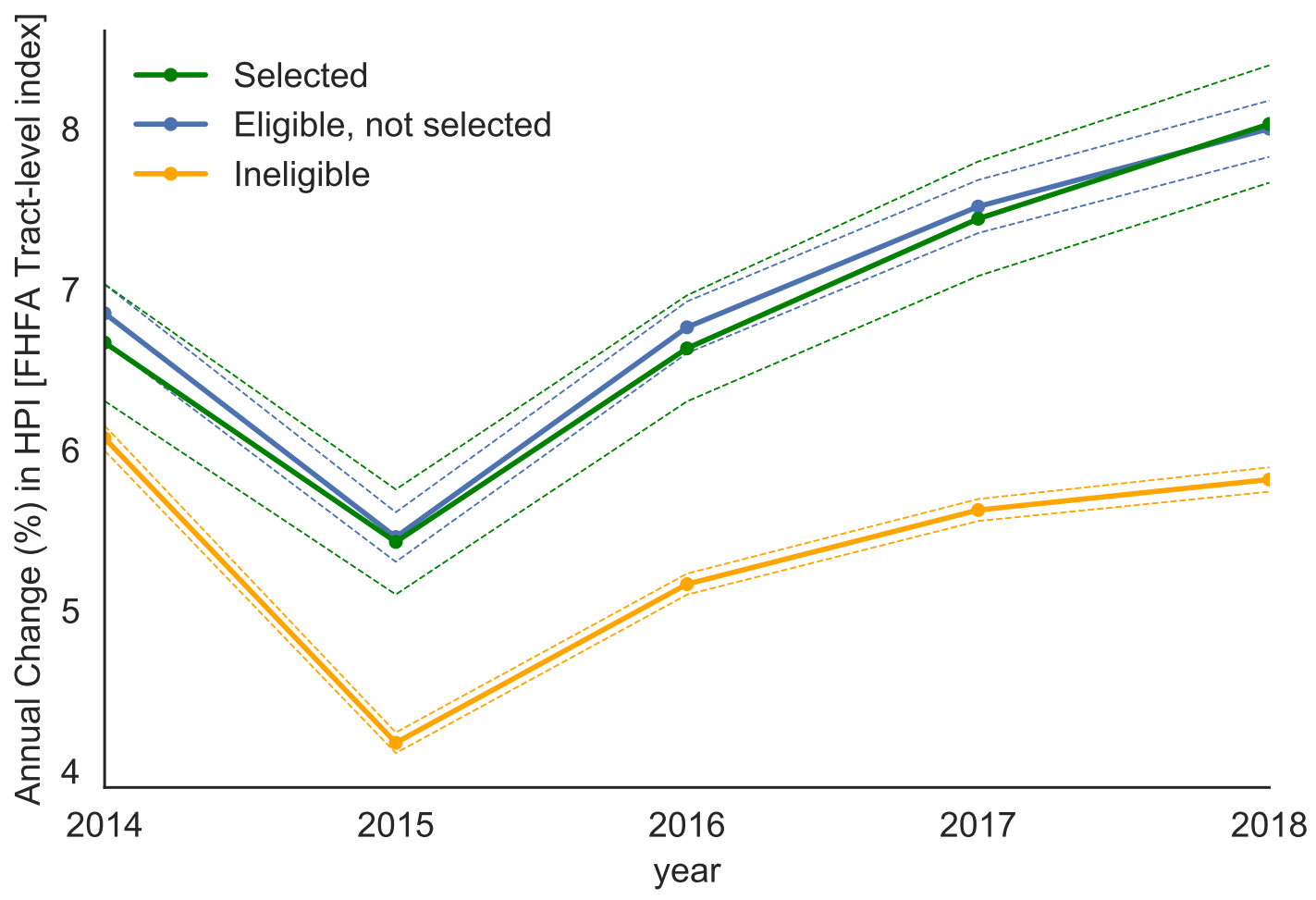

Figure 2: Trend and event study plot for top panel of Table 1 


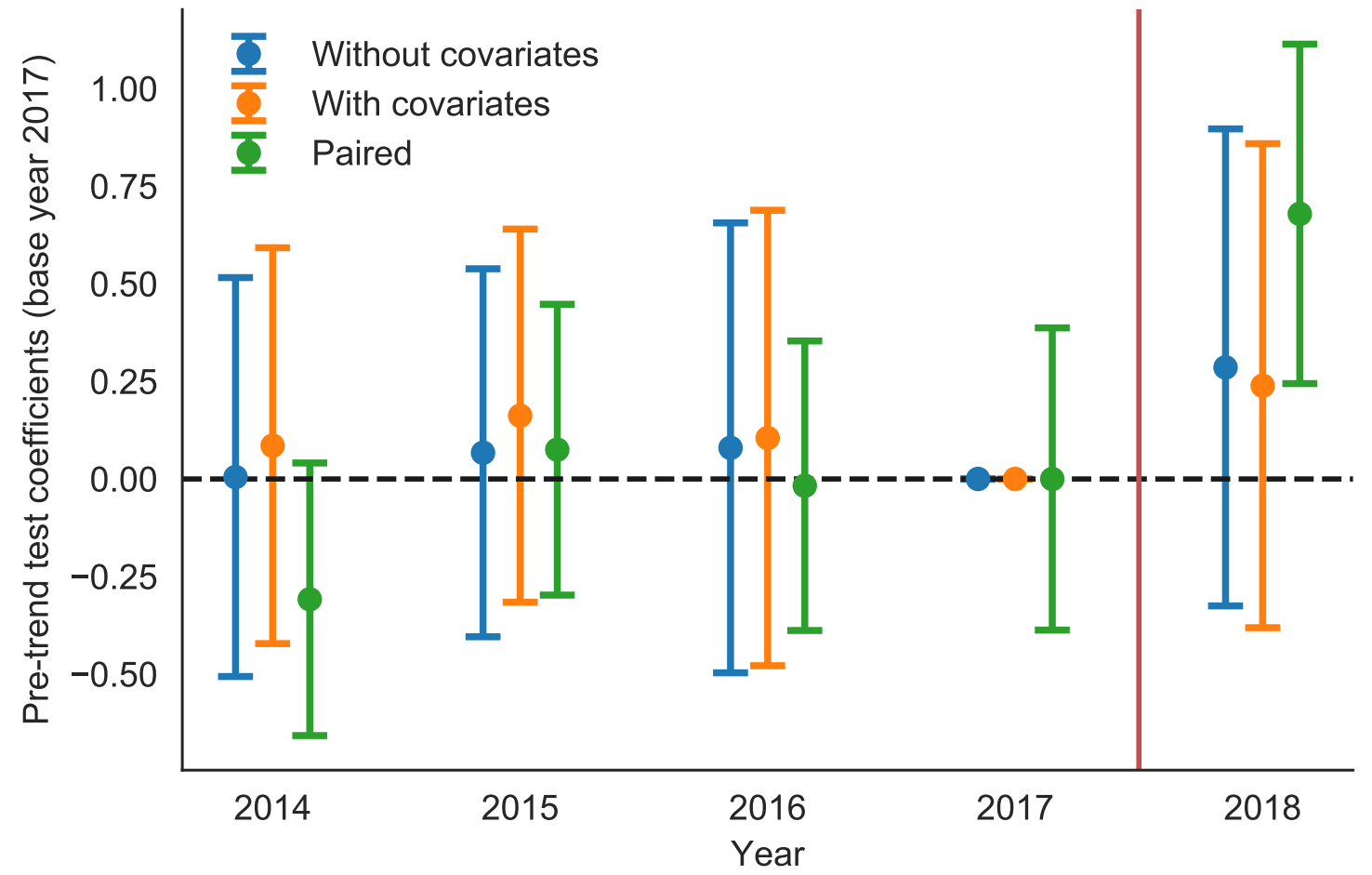

Figure 3: Trend and event study plot for top panel of Table 1 
Table 1: Estimation of ATT using FHFA Tract and ZIP-level data

\begin{tabular}{|c|c|c|c|c|c|c|}
\hline & $\begin{array}{l}\text { TWFE } \\
\text { (1) }\end{array}$ & $\begin{array}{l}\text { TWFE } \\
(2)\end{array}$ & $\begin{array}{c}\text { Weighting CS } \\
\text { (3) }\end{array}$ & $\begin{array}{c}\text { Weighting DR } \\
\text { (4) }\end{array}$ & $\begin{array}{l}\text { Paired } \\
\quad(5)\end{array}$ & $\begin{array}{l}\text { Paired } \\
\quad(6)\end{array}$ \\
\hline \multicolumn{7}{|l|}{ Tract-level data } \\
\hline$\widehat{\tau}$ & 0.248 & 0.151 & 0.254 & 0.085 & 0.742 & 0.534 \\
\hline & $(0.221)$ & $(0.222)$ & $(0.323)$ & $(0.328)$ & $(0.249)$ & $(0.355)$ \\
\hline$p$-value & 0.261 & 0.497 & 0.431 & 0.797 & 0.003 & 0.133 \\
\hline Pre-trend test $p$-value & 0.987 & 0.926 & 0.851 & - & 0.510 & - \\
\hline$\left(N_{1}, N_{0}\right)$ & $(2674,10198)$ & $(2674,10198)$ & $(2674,10198)$ & $(2863,10795)$ & $(2661,2661)$ & $(2661,2661)$ \\
\hline Covariates & No & Yes & Yes & Yes & - & - \\
\hline Sample & Balanced (2014-2018) & Balanced (2014-2018) & Balanced (2014-2018) & Balanced (2017-2018) & Paired (2014-2018) & Paired (2014-2018) \\
\hline Trend & - & - & - & - & None & Linear \\
\hline Model & Within & Within & Weighting & Weighting & Within & Within \\
\hline \multicolumn{7}{|l|}{ ZIP-level data } \\
\hline$\widehat{\tau}$ & 0.477 & -0.306 & & -0.610 & & \\
\hline & $(0.412)$ & $(0.455)$ & & $(0.438)$ & & \\
\hline$p$-value & 0.247 & 0.501 & & 0.163 & & \\
\hline Pre-trend test $p$-value & 0.020 & 0.676 & & - & & \\
\hline$N$ & 11,583 & 11,583 & & $(1084,8534)$ & & \\
\hline Covariates & No & Yes & & Yes & & \\
\hline Sample & Unbalanced (2014-2018) & Unbalanced (2014-2018) & & Balanced (2017-2018) & & \\
\hline Model & Within & Within & & Weighting & & \\
\hline
\end{tabular}

Covariates include log median household income, total housing units, percent white, percent with post-secondary education, percent rental units, percent covered by health insurance among native-born individuals, percent below poverty line, percent receiving supplemental income, and percent employed. For Column (2), only including log median household income and percent white as covariates gives 0.033 (0.222) for the top panel and -0.379 (0.439) for the bottom panel.

Pretest for Column (2) interacts covariates with time dummies. Discrete treatment in Column (4) is defined as the highest $88.3 \%$ of treated tract coverage, so as to keep the percentage of treated ZIPs the same as treated tracts. 
Table 2: By percent employment population quartile

\begin{tabular}{llcc}
\hline & & $\begin{array}{c}\text { No Covariates } \\
(1)\end{array}$ & $\begin{array}{c}\text { Covariates } \\
(2)\end{array}$ \\
\hline \hline Quartile 1 & $\widehat{\tau}$ & -1.189 & -1.721 \\
& & $(0.949)$ & $(1.034)$ \\
& $p$-value & 0.210 & 0.096 \\
& Pre-trend test $p$-value & 0.768 & 0.664 \\
& $N$ & 2,878 & 2,878 \\
\hline Quartile 2 & $\widehat{\tau}$ & 0.583 & -0.067 \\
& & $(0.872)$ & $(0.942)$ \\
& $p$-value & 0.504 & 0.943 \\
& Pre-trend test $p$-value & 0.448 & 0.836 \\
& $N$ & 2,892 & 2,892 \\
\hline \multirow{2}{*}{ Quartile 3} & $\widehat{\tau}$ & 1.940 & 1.149 \\
& & $(0.768)$ & $(0.848)$ \\
& $p$-value & 0.012 & 0.175 \\
& Pre-trend test $p$-value & 0.054 & 0.723 \\
& $N$ & 2,891 & 2,891 \\
\hline \multirow{2}{*}{ Quartile 4} & $\widehat{\tau}$ & 0.679 & -0.705 \\
& & $(0.699)$ & $(0.809)$ \\
& $p$-value & 0.331 & 0.383 \\
& Pre-trend test $p$-value & $6.656 \times 10^{-5}$ & 0.057 \\
& $N$ & 2,892 & 2,892 \\
\hline
\end{tabular}




\section{References}

Abadie, A. (2005). Semiparametric difference-in-differences estimators. The Review of Economic Studies 72(1), 1-19.

Abraham, S. and L. Sun (2018). Estimating dynamic treatment effects in event studies with heterogeneous treatment effects. Available at SSRN $315874 \%$.

Angrist, J. D. and J.-S. Pischke (2008). Mostly harmless econometrics: An empiricist's companion. Princeton university press.

Athey, S. and G. W. Imbens (2018). Design-based analysis in difference-in-differences settings with staggered adoption. Technical report, National Bureau of Economic Research.

Borusyak, K. and X. Jaravel (2017). Revisiting event study designs. Available at SSRN 2826228.

Busso, M. and P. Kline (2008). Do local economic development programs work? evidence from the federal empowerment zone program.

Callaway, B. and P. H. Sant'Anna (2018). Difference-in-differences with multiple time periods and an application on the minimum wage and employment. arXiv preprint arXiv:1803.09015.

Casey, A. (2019). Sale prices surge in neighborhoods with new tax break. https://www.zillow. com/research/prices-surge-opportunity-zones-23393/. Accessed: 2019-11-14.

De Chaisemartin, C. and X. D'Haultfœuille (2017). Fuzzy differences-in-differences. The Review of Economic Studies 85(2), 999-1028.

Goodman-Bacon, A. (2018). Difference-in-differences with variation in treatment timing. Technical report, National Bureau of Economic Research.

Imai, K. and I. S. Kim (2016). When should we use linear fixed effects regression models for causal inference with longitudinal data? Unpublished working paper. Retrieved September 19, 2018.

Neumark, D. and J. Kolko (2010). Do enterprise zones create jobs? evidence from california's enterprise zone program. Journal of Urban Economics 68(1), 1-19.

Sant'Anna, P. H. and J. B. Zhao (2018). Doubly robust difference-in-differences estimators. Available at SSRN 3293315. 


\section{A Covariates, summary statistics, and balance}

Variable definitions for covariates and simple calculations, along with their associated code used in the Census API, are shown in Table 3. Summary statistics and covariate balance are shown in Tables 4 and 5. We see that compare to the control group, the selected OZs are less populated, less employed, less likely to attain higher education, have more rental units, and are less wealthy. Similar trends persist when compared to their non-selected geographical neighbors in Table 5. While the covariate non-balance threatens identification by making the (conditional) parallel trends assumption less plausible, it does not reject parallel trends either. Since identification requires only trends to be parallel and allows for level differences, it also allows for level differences in observed or unobserved characteristics, so long as the trends are the same (conditionally).

\section{B Simple parametric design}

We also include a simple parametric design by regression the time-difference of housing price growth (2018 minus 2017) on treatment status and on covariates. We present the results in Table 6. The estimated treatment effects are similar to those in the main text.

\section{Including all Opportunity Zones}

In the main text, we compare treated zones to control zones that are strictly low-income. We remove this restriction in this section. We regenerate Figures 2 and 3 with Figure 4, Table 1 with Table 7, and Table 2 with Table 8 . The modification does not change our qualitative results. 
Table 3: Variable definitions, ACS codes, descriptions, and transformations

\begin{tabular}{|c|c|}
\hline & $\begin{array}{c}\text { description } \\
\text { (1) }\end{array}$ \\
\hline B01003_001E & population \\
\hline B02001_002E & white_population \\
\hline C24020_001E & employed_population \\
\hline B08131_001E & minutes_commute \\
\hline B09010_002E & supplemental_income \\
\hline B15003_021E & associate \\
\hline B15003_022E & bachelor \\
\hline B15003_023E & master \\
\hline B15003_024E & professional_school \\
\hline B15003_025E & doctoral \\
\hline B16009_002E & poverty \\
\hline B18140_001E & median_earnings \\
\hline B19019_001E & median_household_income \\
\hline B25011_001E & total_housing \\
\hline B25011_026E & renter_occupied \\
\hline B25031_001E & median_gross_rent \\
\hline B27020_002E & native_born \\
\hline B27020_003E & native_born_hc_covered \\
\hline pct_white & white_population / population \\
\hline minutes_commute & minutes_commute / employed_population \\
\hline $\begin{array}{l}\text { pct_higher_ed } \\
\text { pct_rent }\end{array}$ & $\begin{array}{c}\text { (associate }+ \text { bachelor }+ \text { professional_school + doctoral) / population } \\
\text { renter_occupied / total_housing }\end{array}$ \\
\hline pct_native_hc_covered & native_born_hc_covered / native_born \\
\hline pct_poverty & poverty / population \\
\hline log_median_earnings & $\log$ (median_earnings) \\
\hline log_median_household_income & $\log ($ median_household_income) \\
\hline log_median_gross_rent & $\log \left(m e d i a n \_g r o s s \_r e n t\right)$ \\
\hline pct_supplemental_income & supplemental_income / population \\
\hline pct_employed & employed_population / population \\
\hline
\end{tabular}


Table 4: Balance of selected opportunity zones and eligible census tracts

\begin{tabular}{lcccccc}
\hline & \multicolumn{2}{c}{ Mean } & Diff. & \multicolumn{2}{c}{ SE } & $t$ \\
& Not Selected & Selected & & Not Selected & Selected & $(5)$ \\
& $(1)$ & $(2)$ & $(3)$ & $(4)$ & $(6)$ \\
\hline Population & 4084.088 & 4018.494 & -65.594 & 12.424 & 22.582 & -2.545 \\
Employed pop. & 1197.027 & 1085.639 & -111.388 & 4.288 & 7.356 & -13.082 \\
Avg. commute (min) & 37.864 & 37.190 & -0.675 & 0.135 & 0.223 & -2.590 \\
Median household income & 26076.123 & 24150.852 & -1925.272 & 47.183 & 81.187 & -20.503 \\
Median earnings & 41606.969 & 36041.271 & -5565.698 & 86.632 & 145.624 & -32.847 \\
Total housing & 1478.728 & 1458.415 & -20.313 & 4.352 & 7.820 & -2.270 \\
Median gross rent & 897.552 & 822.828 & -74.724 & 1.953 & 3.053 & -20.616 \\
\% White & 0.624 & 0.568 & -0.057 & 0.002 & 0.003 & -14.558 \\
\% Higher ed. & 0.144 & 0.129 & -0.014 & $4.790 \times 10^{-4}$ & $7.741 \times 10^{-4}$ & -15.677 \\
\% Rent & 0.490 & 0.557 & 0.067 & 0.001 & 0.003 & 22.981 \\
\% Healthcare & 0.886 & 0.878 & -0.007 & $3.976 \times 10^{-4}$ & $7.260 \times 10^{-4}$ & -9.043 \\
\% Poverty & 0.207 & 0.249 & 0.043 & $6.371 \times 10^{-4}$ & 0.001 & 30.347 \\
\% Supplemental income & 0.101 & 0.120 & 0.019 & $4.143 \times 10^{-4}$ & $8.284 \times 10^{-4}$ & 20.923 \\
\% Employed & 0.290 & 0.266 & -0.024 & $5.056 \times 10^{-4}$ & $8.789 \times 10^{-4}$ & -23.720 \\
\hline
\end{tabular}

"Not Selected" refers to eligible but not selected opportunity zones. Difference is selected minus not selected. Two-sample $t$-statistic reported. 
Table 5: Covariate balance between geographical pairs (treated minus untreated)

\begin{tabular}{lcccc}
\hline & $N$ & Mean & Standard Err. & $t$-statistic \\
& $(1)$ & $(2)$ & $(3)$ & $(4)$ \\
\hline Population & 7,814 & -639.104 & 28.431 & -22.479 \\
Employed pop. & 7,814 & -423.939 & 9.933 & -42.681 \\
Avg. commute (min) & 3,259 & 2.234 & 0.230 & 9.710 \\
Median household income & 7,797 & -7303.578 & 119.613 & -61.060 \\
Median earnings & 7,800 & -18271.712 & 231.782 & -78.831 \\
Total housing & 7,814 & -269.094 & 10.282 & -26.172 \\
Median gross rent & 7,747 & -136.712 & 3.111 & -43.940 \\
\% White & 7,814 & -0.136 & 0.003 & -49.649 \\
\% Higher ed. & 7,814 & -0.062 & 0.001 & -58.423 \\
\% Rent & 7,808 & 0.175 & 0.003 & 69.359 \\
\% Healthcare & 7,813 & -0.028 & $6.960 \times 10^{-4}$ & -40.157 \\
\% Poverty & 7,814 & 0.101 & 0.001 & 74.251 \\
\% Supplemental income & 7,814 & 0.050 & $8.875 \times 10^{-4}$ & 55.862 \\
\% Employed & 7,814 & -0.061 & 0.001 & -58.437 \\
\hline
\end{tabular}

Table 6: Simple parametric specification

\begin{tabular}{lccc}
\hline & $\begin{array}{c}\text { Short OLS } \\
(1)\end{array}$ & $\begin{array}{c}\text { Long OLS } \\
(2)\end{array}$ & $\begin{array}{c}\text { Selection OLS } \\
(3)\end{array}$ \\
\hline Selected as OZ & 0.248 & 0.151 & \\
& $(0.210)$ & $(0.212)$ & -0.116 \\
Log median household income & & -5.164 & $(0.021)$ \\
& & $(0.502)$ & -0.475 \\
\% Employed & 6.757 & $(0.073)$ \\
\% Post-secondary & $(1.765)$ & 0.004 \\
& & 1.340 & $(0.069)$ \\
\% Healthcare & $(1.670)$ & 0.144 \\
& & 4.418 & $(0.076)$ \\
\% Poverty & $(1.841)$ & 0.155 \\
\% Rental & & -4.496 & $(0.070)$ \\
\% Supplemental Income & $(1.691)$ & 0.151 \\
& & -3.746 & $(0.025)$ \\
\% White & & $(0.614)$ & 0.232 \\
& & -1.131 & $(0.093)$ \\
Total housing units & & $(2.237)$ & 0.042 \\
& & -0.612 & $(0.018)$ \\
$N$ & & $(0.425)$ & $2.914 \times 10^{-5}$ \\
$R^{2}$ & & $4.912 \times 10^{-4}$ & $\left(12.680 \times 10^{-6}\right)$ \\
Sample & & $\left(1.369 \times 10^{-4}\right)$ & 12,872 \\
\end{tabular}


Table 7: Estimation of ATT using FHFA Tract and ZIP-level data

\begin{tabular}{|c|c|c|c|c|c|c|}
\hline & $\begin{array}{l}\text { TWFE } \\
(1)\end{array}$ & $\begin{array}{l}\text { TWFE } \\
(2)\end{array}$ & $\begin{array}{c}\text { Weighting CS } \\
(3)\end{array}$ & $\begin{array}{c}\text { Weighting DR } \\
(4)\end{array}$ & $\begin{array}{c}\text { Paired } \\
(5)\end{array}$ & $\begin{array}{c}\text { Paired } \\
(6)\end{array}$ \\
\hline \multicolumn{7}{|l|}{ Tract-level data } \\
\hline$\widehat{\tau}$ & 0.336 & 0.250 & 0.306 & 0.104 & 0.742 & 0.534 \\
\hline & $(0.206)$ & $(0.212)$ & $(0.325)$ & $(0.324)$ & $(0.249)$ & $(0.355)$ \\
\hline$p$-value & 0.104 & 0.239 & 0.347 & 0.748 & 0.003 & 0.133 \\
\hline Pre-trend test $p$-value & 0.804 & 0.920 & 0.923 & - & 0.510 & - \\
\hline$\left(N_{1}, N_{0}\right)$ & $(2802,17323)$ & $(2802,17323)$ & $(2802,17323)$ & $(2995,18075)$ & $(2661,2661)$ & $(2661,2661)$ \\
\hline Covariates & No & Yes & Yes & Yes & - & - \\
\hline Sample & Balanced (2014-2018) & Balanced (2014-2018) & Balanced (2014-2018) & Balanced (2017-2018) & Paired (2014-2018) & Paired (2014-2018) \\
\hline Trend & - & - & - & - & None & Linear \\
\hline Model & Within & Within & Weighting & Weighting & Within & Within \\
\hline \multicolumn{7}{|l|}{ ZIP-level data } \\
\hline$\widehat{\tau}$ & 0.514 & -0.196 & & -0.492 & & \\
\hline & $(0.393)$ & $(0.436)$ & & $(0.422)$ & & \\
\hline$p$-value & 0.191 & 0.654 & & 0.244 & & \\
\hline Pre-trend test $p$-value & 0.024 & 0.392 & & - & & \\
\hline$N$ & 14,123 & 14,123 & & $(1339,10223)$ & & \\
\hline Covariates & No & Yes & & Yes & & \\
\hline Sample & Unbalanced (2014-2018) & Unbalanced (2014-2018) & & Balanced (2017-2018) & & \\
\hline Model & Within & Within & & Weighting & & \\
\hline
\end{tabular}

Covariates include log median household income, total housing units, percent white, percent with post-secondary education, percent rental units, percent covered by health insurance among native-born individuals, percent below poverty line, percent receiving supplemental income, and percent employed. For Column (2), only including log median household income and percent white as covariates gives 0.033 (0.211) for the top panel and -0.279 (0.419) for the bottom panel.

Pretest for Column (2) interacts covariates with time dummies. Discrete treatment in Column (4) is defined as the highest $88.3 \%$ of treated tract coverage, so as to keep the percentage of treated ZIPs the same as treated tracts. 
Table 8: By percent employment population quartile

\begin{tabular}{llcc}
\hline & & $\begin{array}{c}\text { No Covariates } \\
(1)\end{array}$ & $\begin{array}{c}\text { Covariates } \\
(2)\end{array}$ \\
\hline \hline Quartile 1 & $\widehat{\tau}$ & -1.435 & -2.173 \\
& & $(1.011)$ & $(1.111)$ \\
& $p$-value & 0.156 & 0.050 \\
& Pre-trend test $p$-value & 0.778 & 0.656 \\
& $N$ & 3,508 & 3,508 \\
\hline Quartile 2 & $\widehat{\tau}$ & 1.037 & 0.269 \\
& & $(0.840)$ & $(0.921)$ \\
& $p$-value & 0.217 & 0.770 \\
& Pre-trend test $p$-value & 0.865 & 0.999 \\
& $N$ & 3,527 & 3,527 \\
\hline \multirow{2}{*}{ Quartile 3} & $\widehat{\tau}$ & 2.167 & 1.567 \\
& & $(0.712)$ & $(0.777)$ \\
& $p$-value & 0.002 & 0.044 \\
& Pre-trend test $p$-value & 0.009 & 0.525 \\
& $N$ & 3,526 & 3,526 \\
\hline \multirow{2}{*}{ Quartile 4} & $\widehat{\tau}$ & 0.120 & -1.111 \\
& & $(0.649)$ & $(0.757)$ \\
& $p$-value & 0.854 & 0.142 \\
& Pre-trend test $p$-value & $1.692 \times 10^{-6}$ & 0.012 \\
& $N$ & 3,527 & 3,527 \\
\hline
\end{tabular}



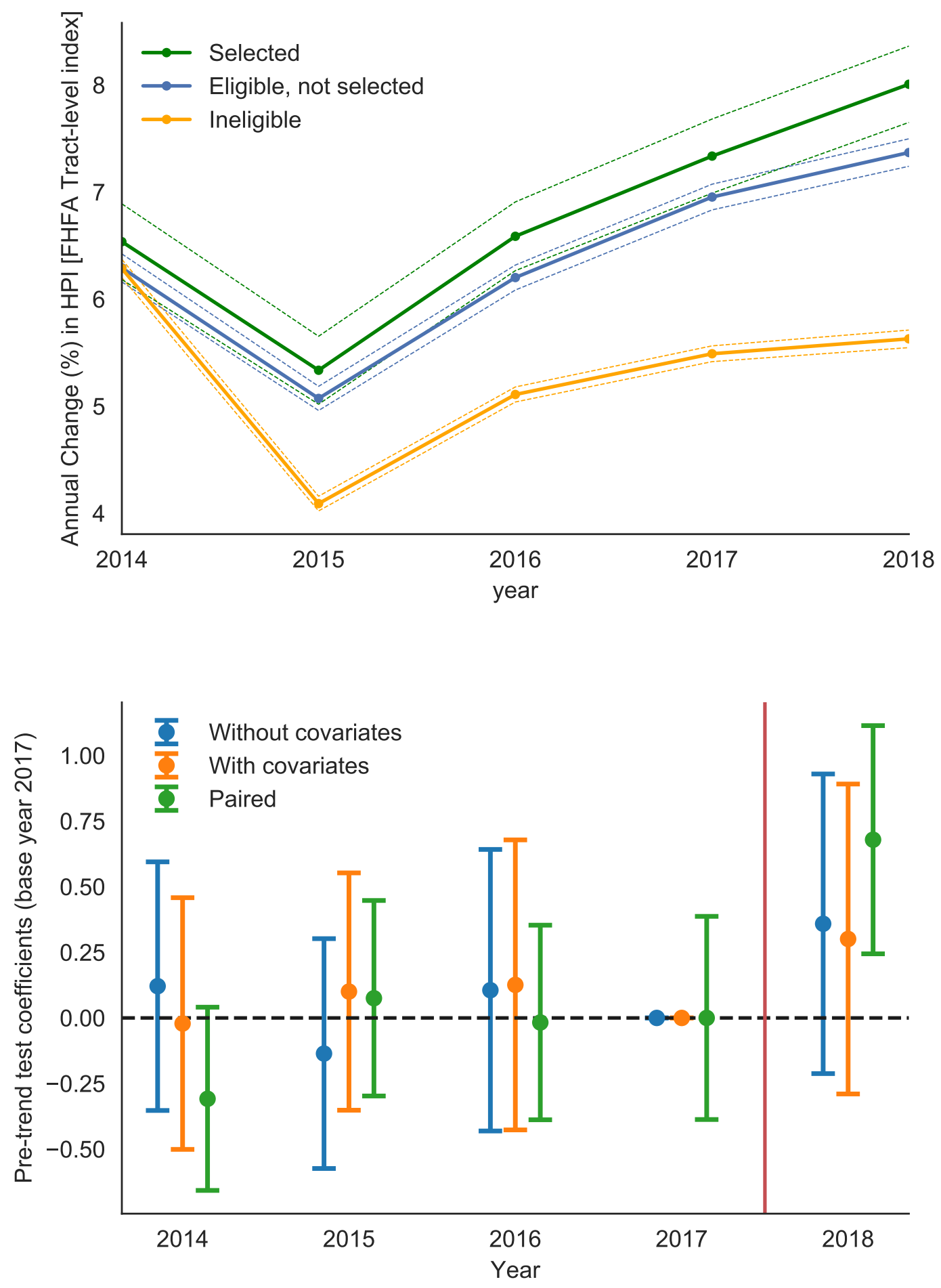

Figure 4: Figures 2 and 3 with all eligible tracts as control group 